\title{
3D WHOLE-PROMINENCE FINE STRUCTURE MODELING. II. PROMINENCE EVOLUTION
}

\author{
Stanislav Gunár ${ }^{1}$ and Duncan H. Mackay \\ School of Mathematics and Statistics, University of St. Andrews, North Haugh, St. Andrews, KY16 9SS, UK \\ Received 2015 May 5; accepted 2015 September 8; published 2015 October 12
}

\begin{abstract}
We use the new three-dimensional (3D) whole-prominence fine structure model to study the evolution of prominences and their fine structures in response to changes in the underlying photospheric magnetic flux distribution. The applied model combines a detailed 3D prominence magnetic field configuration with a realistic description of the prominence plasma distributed along multiple fine structures. In addition, we utilize an approximate $\mathrm{H} \alpha$ visualization technique to study the evolution of the visible cool prominence plasma both in emission (prominence) and absorption (filament). We show that the initial magnetic field configuration of the modeled prominence is significantly disturbed by the changing position of a single polarity of a magnetic bipole as the bipole is advected toward the main body of the filament. This leads to the creation of a barb, which becomes the dominant feature visible in the synthetic $\mathrm{H} \alpha$ images of both the prominence and filament views. The evolution of the bipole also creates conditions that lead to the disappearance and reappearance of large portions of the main body. We also show that an arch-like region containing a dark void (a bubble) can be naturally produced in the synthetic prominence $\mathrm{H} \alpha$ images. While not visible in terms of the magnetic field lines, it is due to a lack of $\mathrm{H} \alpha$ emission from low-pressure, low-density plasma located in shallow magnetic dips lying along the lines of sight intersecting the dark void. In addition, a quasi-vertical small-scale feature consisting of short and deep dips, piled one above the other, is produced.
\end{abstract}

Key words: radiative transfer - Sun: filaments, prominences - Sun: magnetic fields

\section{INTRODUCTION}

To date, research into the evolution of prominences has mainly being carried out either in terms of the prominence's magnetic field (see, e.g., the review by Mackay et al. 2010) or in terms of the observable prominence plasma (see Labrosse et al. 2010). Studies that consider the evolution of the prominence magnetic field mainly use magnetohydrodynamic (MHD) models that evolve an initial magnetic field configuration over consecutive time steps. Such models include van Ballegooijen \& Martens (1989), DeVore \& Antiochos (2000), Galsgaard \& Longbottom (1999), Lionello et al. (2002), Mackay \& Gaizauskas (2003), Mackay \& van Ballegooijen (2005, 2006, 2009), Aulanier et al. (2006), Martens \& Zwaan (2001), and Xia et al. (2011, 2012). These simulations study the prominence evolution mainly in terms of the magnetic field. To simulate the appearance of mass-loaded dips, they often employ a simple technique of drawing portions of field lines at the position of the magnetic dips-a simplified representation of the prominence plasma (see the discussion of this technique in Gunár 2014). This is beneficial for the assessment of the large-scale changes of the prominence structure in connection to the variation of the photospheric flux distribution below it. However, it does not clearly indicate how these changes translate into the changes of the prominence plasma and thus to the changes that are visible in observations at different wavelengths.

In contrast to magnetic field modeling, studies of prominence evolution from observations are not able to directly reveal the prominence magnetic field structure. Only an indirect link exists between the observed evolution of the large- and medium-scale prominence structures seen in a variety of wavelengths and the evolution of the prominence magnetic field configuration. This is even more complicated when the

\footnotetext{
${ }^{1}$ On leave from the Astronomical Institute of the Czech Academy of Sciences.
}

evolution of the prominence's fine structures is taken into account, due to the fact that they depend heavily on the local thermodynamical properties (see, e.g., Labrosse et al. 2010). The dynamics of prominence fine structures has been analyzed by many authors, among them Schmieder et al. (2010), Berger et al. (2011), Gunár et al. (2012), and Heinzel et al. (2015b). For a comprehensive review of prominence dynamics see Berger (2014) and for a review of general prominence properties, see Labrosse et al. (2010). Modeling the dynamics of prominence fine structures as a result of the magnetic Rayleigh-Taylor instability has been carried out by Hillier et al. (2012). The dynamical behavior of prominence fine structure plasma is also considered in the simulations of prominence oscillations - see the review by Ballester et al. (2014). Some studies of prominence fine structures even indicate a dynamical behavior in contradiction to the generally assumed predominantly horizontal prominence magnetic field configurations (Berger et al. 2008, 2010). The only direct evidence of the prominence magnetic field, obtained from observations, is provided by spectropolarimetry (see, e.g., Leroy 1989, pp. 77-113; Casini et al. 2009; Orozco Suárez et al. 2014).

In the present paper we use the first three-dimensional (3D) whole-prominence fine structure (WPFS) model developed by Gunár \& Mackay (2015, hereafter Paper I) to study the largescale, long-term evolution of prominence and filament fine structures with respect to the evolution of the underlying photospheric magnetic flux distribution. We employ the method of Gunár et al. (2013) to fill magnetic dips produced by the 3D non-linear force-free field (NLFF) model of Mackay \& van Ballegooijen (2009) with prominence plasma in hydrostatic equilibrium. The temperature variation of the prominence plasma is described semi-empirically and it includes a prominence-corona transition region (PCTR). We use the fast approximate $\mathrm{H} \alpha$ radiative transfer method of Heinzel et al. (2015a) to produce synthetic $\mathrm{H} \alpha$ images of the 
modeled prominence. This allows us for the first time to consistently follow the link between the changes of the photospheric flux distribution, the prominence magnetic field configuration, the prominence fine structure plasma, and the observable changes in its appearance both as a prominence (in emission) and as a filament (in absorption). In this paper we consider the main large-scale evolution of the prominence and not the small-scale rapid dynamics of the individual prominence fine structures. Following the latter between individual time steps is beyond the scope of the current paper.

A brief overview of the 3D magnetic field configuration and its evolution, together with a description of the prominence plasma distribution and the applied visualization technique, are given in Section 2. The evolution of the modeled prominence is described in detail in Section 3. Sections 4 and 5 give a discussion of the results and our conclusions.

\section{3D WPFS MODEL}

\subsection{Whole-prominence Magnetic Field Configuration}

As in Paper I, we use the 3D NLFF simulations of Mackay \& van Ballegooijen (2009) to produce a time series of realistic prominence magnetic field configurations. As stated in Paper I, our intent is not to demonstrate the validity of these simulations by comparison with observations of prominences and filaments. We simply use these simulations as a readily available data set with which to employ the WPFS modeling. Details of these simulations are described in Section 3 of Paper I and in Mackay \& van Ballegooijen (2009). In the following paragraph we briefly summarize their setup.

The basic photospheric distribution of magnetic flux is that of a magnetic arcade with displaced magnetic flux concentrations (see Figure 1 of Mackay \& van Ballegooijen 2009). The separation of the arcades is chosen to be $120,000 \mathrm{~km}$, with one dimension-less length-scale unit of $60,000 \mathrm{~km}$. This choice is arbitrary, but is taken such that the arcades lie four typical supergranular cells apart. From the photospheric flux distribution a 3D linear force-free field is constructed where the value of $\alpha$ is fixed at $-1.477 \times 10^{-8} \mathrm{~m}^{-1}$. This is sufficiently large to produce a simple regular dipped magnetic field configuration that represents the basic structure (main body) of a filament/ prominence. The value of $\alpha$ that we have chosen is well below the critical value of $-2.14 \times 10^{-8} \mathrm{~m}^{-1}$. This linear force-free field represents the initial condition into which a bipole is inserted (see Section 2.2.3 of Mackay \& van Ballegooijen 2009). The ratio of bipole flux $\left(F_{\mathrm{b}}\right)$ to arcade flux $\left(F_{\mathrm{a}}\right)$ is $F_{\mathrm{b}} / F_{\mathrm{a}}=0.0084$, while the ratio of bipole to filament flux $\left(F_{\mathrm{f}}\right)$ is $F_{\mathrm{b}} / F_{\mathrm{f}}=2.7$. The bipole is therefore very weak compared to the overall arcade, but strong compared to the flux of the filament (i.e., the flux of the initial dipped portion of the magnetic field). In setting up the initial condition we specify the flux of the arcade and the flux of the bipole. These values are set to specify the flux of a decaying active region and that of an ephemeral region. These values are constrained through observations of typical sized regions and are chosen to lie in the middle range of observed values. The filament flux then depends on the force-free parameter $\alpha$ used to compute the initial condition. By varying $\alpha$ we can make the dipped region bigger or smaller. The $\alpha$ is chosen so that we produce a typical length/height and width of a filament. We then compute the filament flux, from the size of the dipped region. Therefore while the arcade and bipole flux are explicitly fixed by us, the filament flux is not.
However, the filament flux is well within ranges found in other simulations and deduced by observations, i.e., that produced by taking the strength of B from Hanle measurements times the filament height and width.

The inner negative polarity of the inserted bipole is then advected toward the main body of the prominence/filament by a boundary flow with a velocity of $0.1 \mathrm{~km} \mathrm{~s}^{-1}$ while the positive polarity is held fixed. As the minority polarity is advected toward the main body, the whole magnetic field configuration is perturbed and evolves through a series of continuous quasi-static NLFF states. This evolution is described by the magneto-frictional technique of van Ballegooijen et al. (2000) and Mackay \& van Ballegooijen (2006).

\subsection{Evolution of the Magnetic Field Configuration}

In the present paper, to follow the evolution of the modeled prominence we consider a time series of seven quasi-static states that we call snapshots. The first snapshot represents the magnetic field configuration soon after the insertion of the bipole $(200,60 \mathrm{~s}$ time steps). The six following snapshots are each taken after 600, $60 \mathrm{~s}$ time steps, i.e., $10 \mathrm{hr}$ apart. We name them SnAPshot 1, 4, 7, 10, 13, 16, and 19. As in Paper I here we consider the magnetic field configurations with the resolution increased from $256^{3}$ to $1280^{3}$ grid points. This allows us to adequately resolve the prominence plasma fine structures with cross-section widths of $1000 \mathrm{~km}$. Each point in the computational domain is tested for the presence of magnetic dips. These occur when the vertical component of the magnetic field satisfies the conditions $B_{z}=0$ and $\boldsymbol{B} . \nabla B_{z}>0$. We consider magnetic field lines passing through all grid points which satisfy this criterion. To overcome the arbitrary selection effects when selecting field lines (see Section 2 of Paper I) we use the multiple randomization method described in Section 3.1. of Paper I that only selects non-overlapping plasma fine structures (named independent field lines). In Figures 3-9 we plot the dipped portions of all independent field lines in each snapshot. Field lines are displayed in projections onto the $x-y$, $x-z$, and $y-z$ planes. The position of the positive (solid red lines) and negative (dashed blue lines) polarity of the inserted bipole is marked by drawn contours, where the contours represent values of $B_{z}$ at levels $\pm 1, \pm 2, \pm 3$, and $\pm 4 \mathrm{G}$.

\subsection{Prominence Fine Structure Plasma Distribution}

To obtain the plasma distribution within the entire prominence for each snapshot we fill the dipped portions of all selected independent field lines with plasma in hydrostatic equilibrium using the method developed by Gunár et al. (2013). This produces individual prominence plasma fine structures with a realistic distribution of the gas pressure and the temperature, including the PCTR. The implementation of this method to prominence magnetic field configurations with large numbers of prominence fine structures is described in detail in Section 3.2 of Paper I.

The local plasma parameters in the individual prominence plasma fine structures depend on the local configuration of the magnetic field and are thus unique for each fine structure. However, we assume a set of input plasma parameters that are identical for all fine structures in all snapshots. Parameters $T_{0}$ the central minimum temperature, $T_{\text {tr }}$-the transition region temperature at the boundary, $\gamma_{\mathrm{al}}$ - the exponent describing the temperature gradient parallel to the field, and $\gamma_{\mathrm{ac}}$-the exponent 
describing the temperature gradient perpendicular to the fieldprescribe the semi-empirical temperature structure with two distinct shapes of the PCTR. These are represented by a narrow region with a steep temperature gradient in the direction perpendicular to the magnetic field lines and a more extended region with a gradual temperature increase in the direction parallel to the field. We assume here $T_{0}=7000 \mathrm{~K}, T_{\mathrm{tr}}=$ $100,000 \mathrm{~K}, \gamma_{\mathrm{al}}=2$, and $\gamma_{\mathrm{ac}}=60$. We further assume the maximum column mass along the magnetic field line in the middle of any prominence plasma fine structure $M_{0}$ to be $1 \times 10^{-4} \mathrm{~g} \mathrm{~cm}^{-2}$ and the transition region gas pressure at the boundary $p_{\text {tr }}$ to be $0.015 \mathrm{dyn} \mathrm{cm}^{-2}$. We also assume the crosssection width of all fine structures to be $1000 \mathrm{~km}$. For more details, including a quantitative description of the fine structures and a discussion of the choice of these values see Paper I.

To define all of the physical quantities for all fine structures, in all of the snapshots we use the same special 3D grid as in Paper I. The use of such a grid is based on the fact that all dipped portions of the magnetic field lines for all snapshots are oriented mostly parallel to the $x$-axis of the computational domain. This allows us to use a lower resolution of $150 \mathrm{~km}$ along the $x$-axis (along the length of the dips) and a higher resolution of $10 \mathrm{~km}$ only in the $y-z$ plane (see Figure 4 of Paper I). We further assume that the cross-sectional planes of all prominence fine structures are parallel to the $y-z$ plane instead of being strictly perpendicular to the local magnetic field vector.

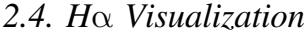

We obtain the synthetic $\mathrm{H} \alpha$ line center images of the modeled prominence/filament through the use of the approximate radiative transfer method for $\mathrm{H} \alpha$ visualization developed by Heinzel et al. (2015a). This method uses fast onedimensional (1D) radiative transfer calculations in the $\mathrm{H} \alpha$ line along any given LOS. It takes into account only the local variation of the temperature and gas pressure along a given line of sight (LOS) intersecting the prominence plasma fine structures. The implementation of this visualization method to the 3D WPFS model is described in detail in Section 4 of Paper I.

In contrast to Paper I, in the present paper we do not assume a constant value of the hydrogen ionization degree $i$ and the factor $f$ describing the relation between the electron density and the hydrogen second-level population (see, e.g., Heinzel et al. 1994). Instead, we interpolate their values from tables given in Heinzel et al. (2015a) based on the local values of the temperature and gas pressure. An altitude of $10,000 \mathrm{~km}$ above the solar surface is assumed everywhere in each snapshot to save computational time. We do not expect significantly different results if the altitude is varied.

To visualize a 3D WPFS model as a prominence seen in emission above the solar limb (i.e., without any background radiation), we use the LOS parallel to the $y$-axis (perpendicular to the $x-z$ plane). This corresponds to the $x-z$ plane view in Figures 3-9. We construct a grid with $150 \times 150 \mathrm{~km}$ spacing that covers the $x-z$ plane to obtain synthetic images with resolutions comparable to observations such as those obtained using the Narrowband Filter Imager (NFI) of the Solar Optical Telescope (SOT, Tsuneta et al. 2008) on board Hinode (Kosugi et al. 2007). The synthetic $\mathrm{H} \alpha$ line center images for each snapshot viewed in the $x-z$ plane are shown in color in
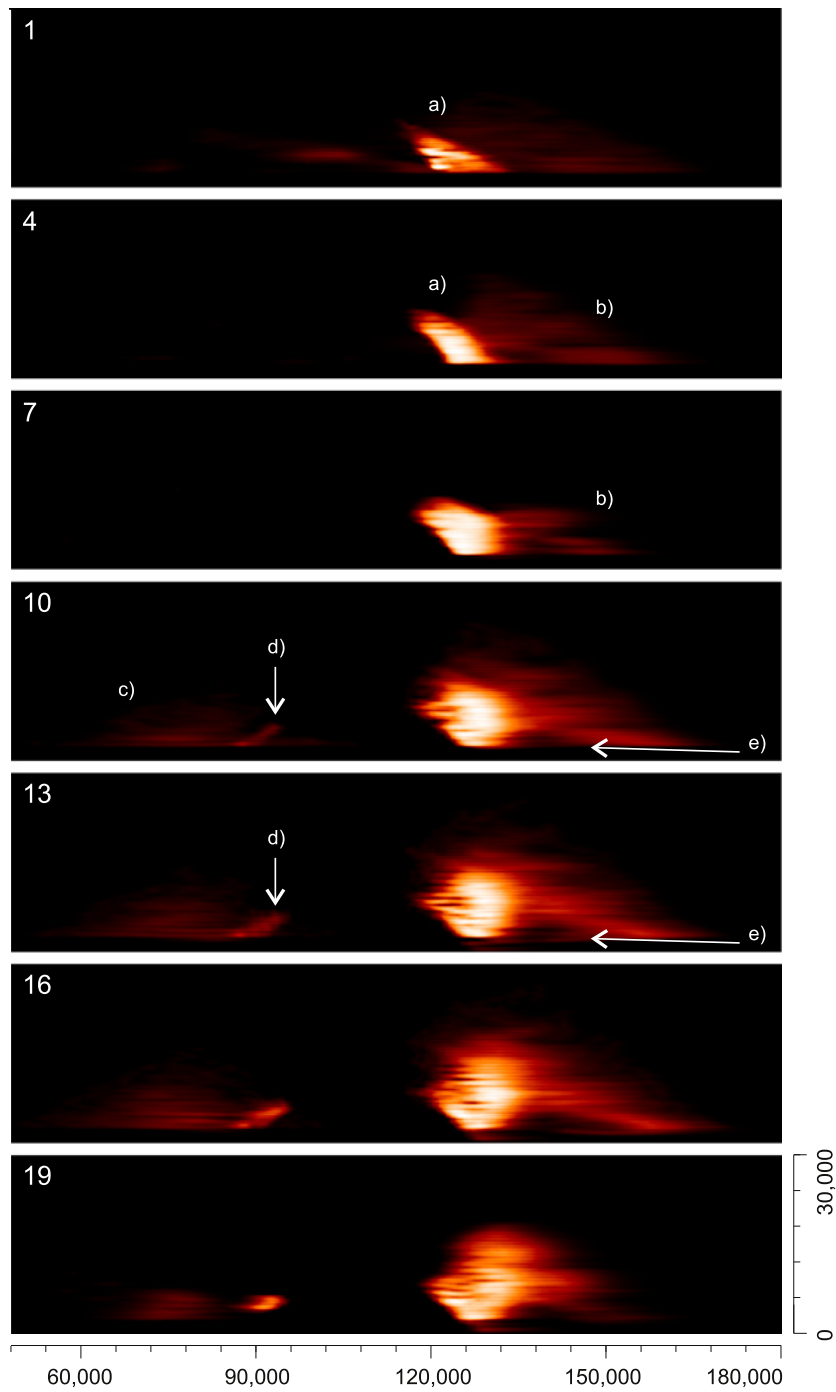

Figure 1. Synthetic prominence $\mathrm{H} \alpha$ images of all snapshots. The intensity scale is indicated in Figures 3-9. Dimensions are in kilometers.

Figure 1 for all snapshots. They are also shown in Figures 3-9 for the individual evolution steps where they can be clearly compared to the drawn dipped portions of the field lines. All prominence images are saturated at a maximum intensity of $3.2 \times 10^{-6} \mathrm{erg} \mathrm{s}^{-1} \mathrm{~cm}^{-2} \mathrm{sr}^{-1} \mathrm{~Hz}^{-1}$.

To visualize a WPFS model as a (dark) filament, i.e., in absorption against the bright solar disk background, we use a LOS that is oriented downward in a direction anti-parallel to the $z$-axis (perpendicular to the $x-y$ plane). This corresponds to the $x-y$ plane view in Figures 3-9. We again construct a grid with $150 \times 150 \mathrm{~km}$ spacing that now covers the $x-y$ plane. The synthetic filament $\mathrm{H} \alpha$ line center images are shown in absorption against the bright solar disk in Figure 2 for all snapshots and also in Figures 3-9 for the individual evolution steps. The intensity of the solar disk $\left(6.93 \times 10^{-6} \mathrm{erg}\right.$ $\mathrm{s}^{-1} \mathrm{~cm}^{-2} \mathrm{sr}^{-1} \mathrm{~Hz}^{-1}$ ) is taken from David (1961). All filament images are saturated at a minimum value of $3.25 \times 10^{-6} \mathrm{erg} \mathrm{s}^{-1} \mathrm{~cm}^{-2} \mathrm{sr}^{-1} \mathrm{~Hz}^{-1}$.

\section{PROMINENCE AND FILAMENT EVOLUTION}

In this section we describe in detail the evolution of the modeled prominence magnetic field and its appearance in the 

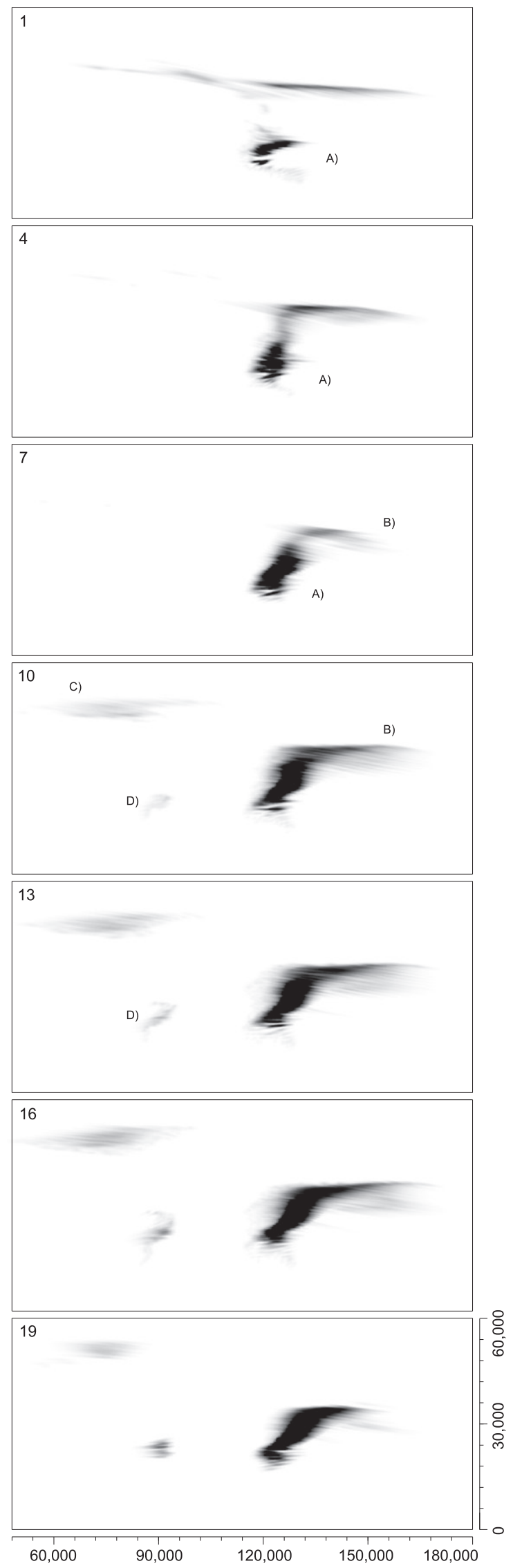

Figure 2. Same as Figure 1 but showing synthetic filament $\mathrm{H} \alpha$ images. synthetic $\mathrm{H} \alpha$ images both as a prominence and as a filament. The evolution is followed at discrete intervals given by the individual snapshots. The same time series was studied in Mackay \& van Ballegooijen (2009), but with a much lower resolution and only the magnetic field was investigated. In Figures 1 and 2 we show the complete time series of snapshots both as a prominence and filament, respectively. Lower-case letters denote individual prominence features in Figure 1 and upper-case letters the corresponding filament features in Figure 2. In Figures 3-9 we show for each snapshot the plots of dipped portions of all independent field lines in the top view ( $x-y$ plane) and side views ( $x-z$ and $y-z$ planes). We also show the synthetic $\mathrm{H} \alpha$ images of the model as a filament and as a prominence. The position of the positive (solid red contours) and negative (dashed blue contours) polarity of the inserted bipole is also marked. We note that the stated depth of the magnetic dips in particular regions described in the following paragraphs can be discerned from the plots showing the dipped portions of the field lines when these plots are magnified in the electronic version of this paper. Examples of such an enlarged view are shown in Figure 10.

SNAPSHOT 1 (Figure 3). From the plots of the dipped magnetic field lines it can be seen that the insertion of the bipole breaks the symmetry of the original arcade and creates a series of shorter but deeper dips between the main body of the prominence/filament and the bipole (see the $x-y$ and the $x-z$ plane panels of Figure 3). These dips are branching out from the main body toward the bipole and are indicative of a barb. The main body consists of a large number of long but relatively shallow highly sheared dips. The spatial volume occupied by the magnetic dips forming the main body is much larger than that taken up by the dips near the inserted bipole. However, this description completely changes when we consider the synthetic $\mathrm{H} \alpha$ images. From the synthetic $\mathrm{H} \alpha$ images we can see that while the main body is faintly visible both as a prominence and filament, the brightest part of the prominence (a) and the darkest part of the filament (A) are composed of short dips formed due to the presence of the bipole. The brightest part of the prominence forms a relatively compact seemingly vertical feature (a). This is, however, composed of numerous horizontal fine structures that align one above the other. The visible main body in the filament image consists of long highly sheared parallel fibrils forming an apparent spine. The region branching out toward the bipole is clearly separated from the main body. This is formed by a series of short dark fibrils following the local magnetic field configuration.

SNAPSHOT 4 (Figure 4). This snapshot is taken $10 \mathrm{hr}$ later, which translates to an advancement of the inner negative polarity toward the main body by $3600 \mathrm{~km}$. While the difference in the magnetic field configuration does not seem dramatic, the observable cool prominence plasma in the synthetic $\mathrm{H} \alpha$ images shows significant changes. The left part of the main body has completely disappeared from the synthetic $\mathrm{H} \alpha$ filament image, even though the magnetic field configuration still shows a large number of dips at this location (see the $x-z$ plane panel of Figure 4). The short deeper dips that branch out toward the bipole (A) are now attached to the right part of the main body. This is seen both in the plot of the dipped field lines and the synthetic $\mathrm{H} \alpha$ filament image. These short deeper dips now form a more uniform barb with mainly 

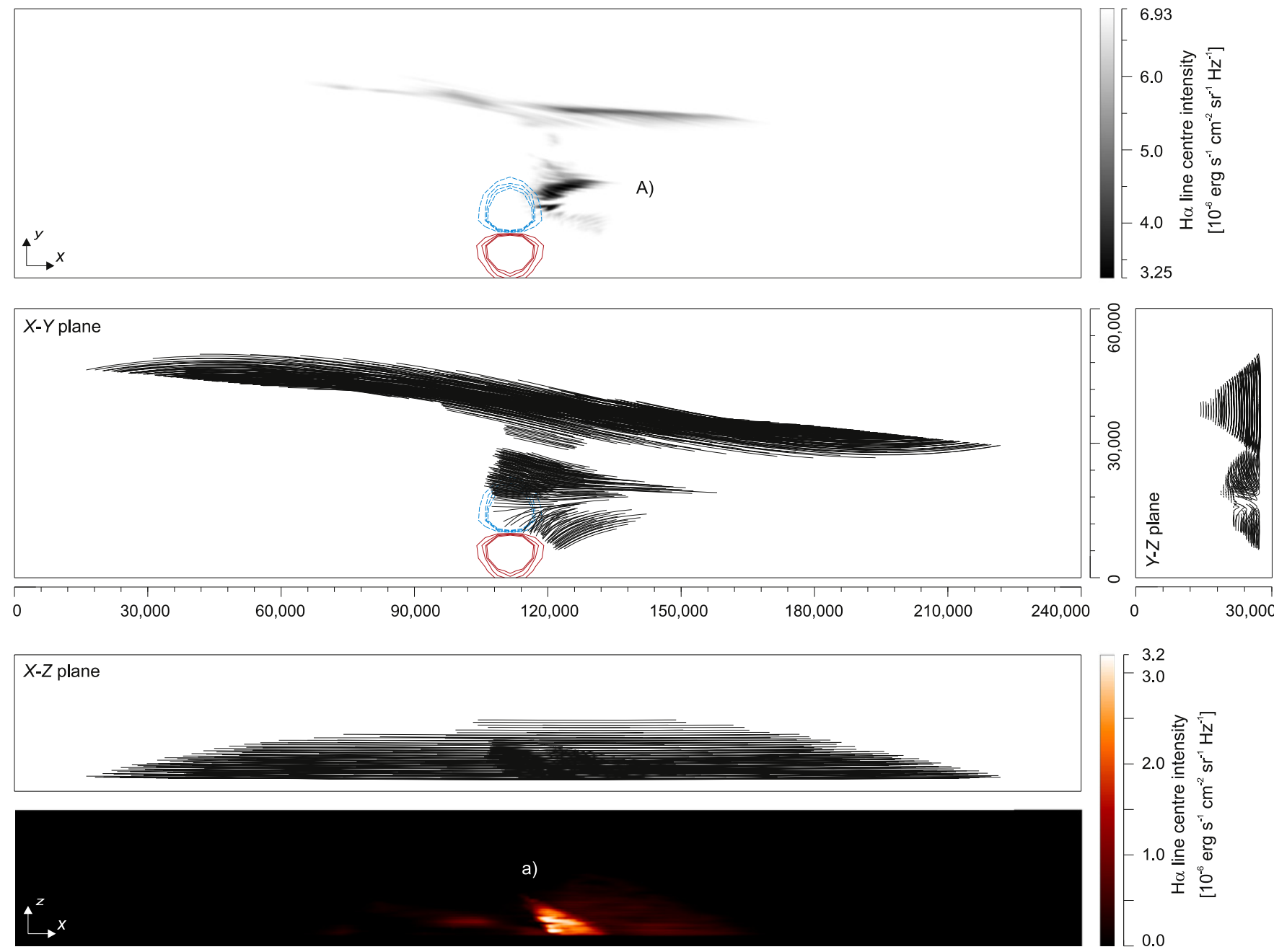

Figure 3. Snapshot 1. Dipped portions of all independent field lines are plotted in the top view ( $x-y$ plane) and side views $(x-z$ and $y-z$ planes). The synthetic H $\alpha$ image of the model as a filament ( $x-y$ plane view) is plotted at the top and as a prominence ( $x-z$ plane view) at the bottom. The position of the positive (solid red lines) and negative (dashed blue lines) polarity of the inserted bipole is marked by drawn contours. Dimensions are in kilometers. (a) denotes the brightest part of the prominence and (A) denotes the darkest part of the filament. These are composed of short dips formed due to the presence of the bipole.

parallel short fibrils that form the darkest region in the filament image. This dark region is caused mainly by the depth of the individual dips (and thus the resulting higher gas pressure) and not by a pile-up of multiple dips one above each other. The synthetic $\mathrm{H} \alpha$ image of the prominence has also changed, compared to the previous snapshot. The central brightest quasivertical feature (a) is now more compact. The right part of the main body (b) is still faintly visible, but the left part has completely disappeared. The central brightest region is visibly formed by horizontal fine structures stacked one above the other. These correspond to the fibrils forming the barb.

SNAPSHOT 7 (Figure 5). After an additional $10 \mathrm{hr}$ and $3600 \mathrm{~km}$ shift of the negative polarity the right side of the main body (B) has also nearly disappeared from the synthetic $\mathrm{H} \alpha$ filament image. In contrast, this is not at all apparent from the plotted field lines, where both parts of the main body have barely changed. In fact, the volume taken up by all magnetic dips even appears to have grown compared to the previous snapshot. The image of the filament now essentially shows only a barb (A) consisting of a series of short dark nearly parallel fibrils. Interestingly, while there is a significant change in the appearance of the filament, the synthetic $\mathrm{H} \alpha$ image of the prominence has not changed much. The central bright compact vertical structure is slightly broader, especially at higher altitudes. The bright horizontal fine structures forming it correspond to the dark fibrils in the filament image. The right part of the main body (b) is faintly visible.

SNAPSHOT 10 (Figure 6). Another shift of the negative polarity toward the main body by $3600 \mathrm{~km}$ causes the magnetic field configuration to split. In the images showing the dipped magnetic field lines the left part of the main body separates from the right and an additional small region of short dips forms on the left of the bipole. In the synthetic filament $\mathrm{H} \alpha$ image the now separated left part (C) becomes faintly visible again compared to the previous snapshot. We discuss the possible causes of this behavior in Section 4. The right part of the main body (B) also becomes visible again, and now appears much broader and darker, formed by long fibrils. The region of short dips on the left (D) is also faintly visible. In the prominence image the brightest part becomes wider and more structured than before. The right part of the main body now forms a clearly visible large halo to the right of the central bright feature. The left part of the main body (c) becomes visible again, albeit faintly. The newly formed small region on the left (d) forms an interesting quasi-vertical feature that can be clearly seen rising from the left part of the prominence 

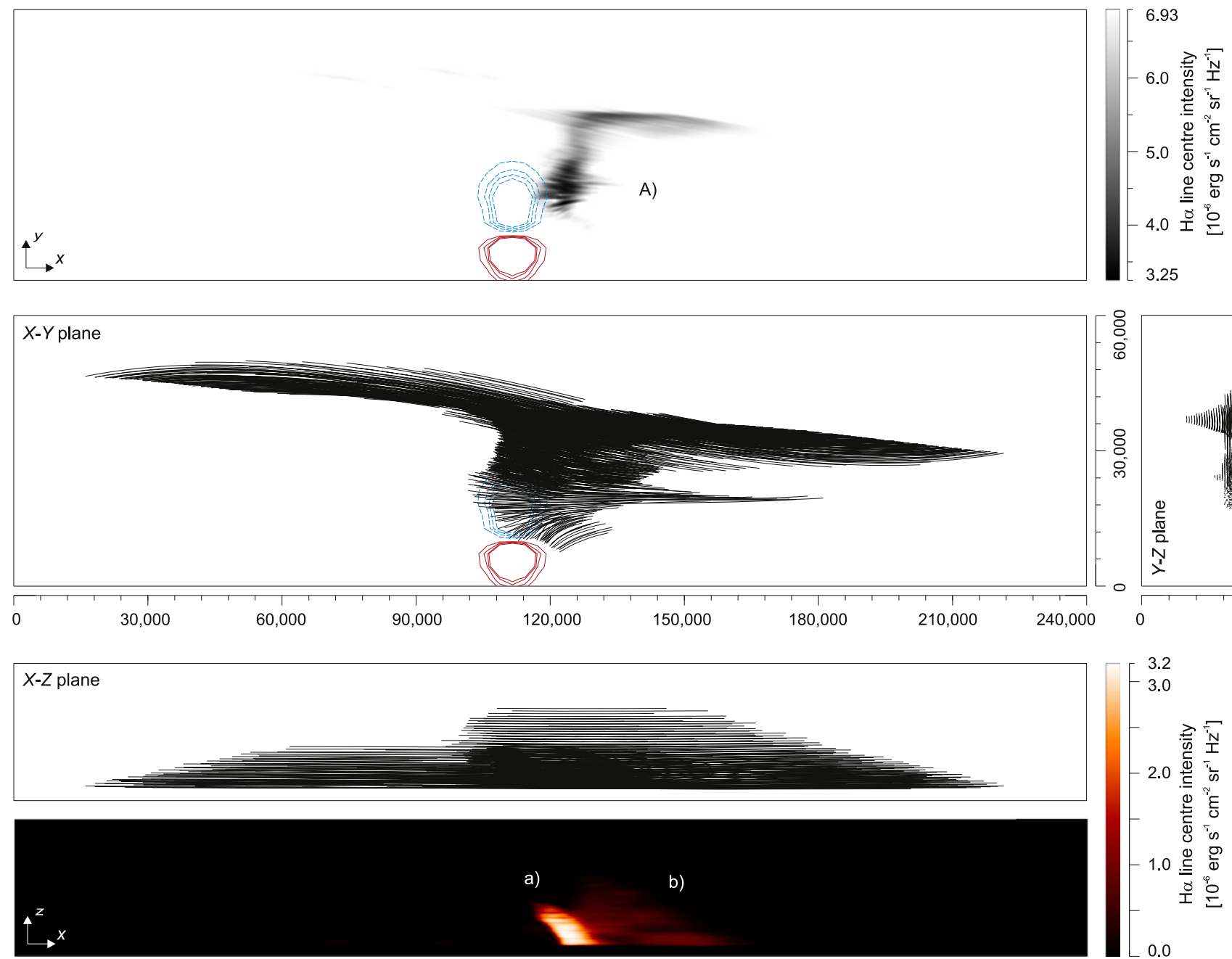

Figure 4. SNAPSHOT 4. Same as in Figure 3. (a) denotes the brightest part of the prominence and (A) denotes the darkest part of the filament. These are composed of short dips formed due to the presence of the bipole. (b) highlights the position of the faintly visible right part of the main body.

toward the bright central part at around a $45^{\circ}$ angle from the vertical. This feature is formed by a pile-up of the short horizontal fine structures staggered one above the other. Interestingly, a darker arch-like region (e) that is devoid of bright emission (but not of magnetic dips) starts to appear on the right, below the central bright feature. This region is not visible in the synthetic filament $\mathrm{H} \alpha$ image.

SNAPSHOT 13 (Figure 7). After another $10 \mathrm{hr}$ the separation of the left part of the main body is more pronounced and is now visible in all three plots of the magnetic field lines. Both the filament and the prominence $\mathrm{H} \alpha$ images appear similar to the previous snapshot, with only the relative intensity changed. The only notable variations are that the small quasi-vertical feature on the left $(\mathrm{d}, \mathrm{D})$ and the dark arch-like region on the right (e) are more clearly visible.

SNAPSHOT 16 (Figure 8). This snapshot is again similar to the previous two. As the negative polarity passes below the former location of the main body the separation of its left part is even wider compared to the previous snapshot. The filament and prominence images are similar to the previous two snapshots. The most significant change is that the dark void on the right below the central bright part of the prominence is now more clearly visible. The void is now clearly surrounded by an area of bright emission and resembles the bubble-like features reported in prominences by Berger et al. $(2010,2011)$ and studied by, e.g., Dudík et al. (2012) or Gunár et al. (2014b). Interestingly, such an arch-like region cannot be identified in the magnetic field configuration itself (see the $x-z$, plane panel of Figure 8) but is clearly visible in the synthetic $\mathrm{H} \alpha$ image.

SNAPSHOT 19 (Figure 9). Another shift of the negative polarity by $3600 \mathrm{~km}$ causes both the right and left parts of the main body to diminish in size again in the filament synthetic $\mathrm{H} \alpha$ image. However, the magnetic field configuration does not change so dramatically. In the prominence $\mathrm{H} \alpha$ image the central bright feature is now tilted in the opposite direction toward the right side (for explanation of this behavior see Section 4). The dark arch-like void becomes smaller and the quasi-vertical feature on the left appears brighter than before, although less extended.

\section{DISCUSSION}

From the presented analysis of the modeled prominence evolution it is evident that even a small change in the underlying photospheric magnetic flux distribution, equivalent to a shift of a single minority polarity by a few thousand kilometers, can cause a large change in the appearance of the prominence. While the evolving photospheric flux distribution significantly affects the prominence magnetic field 

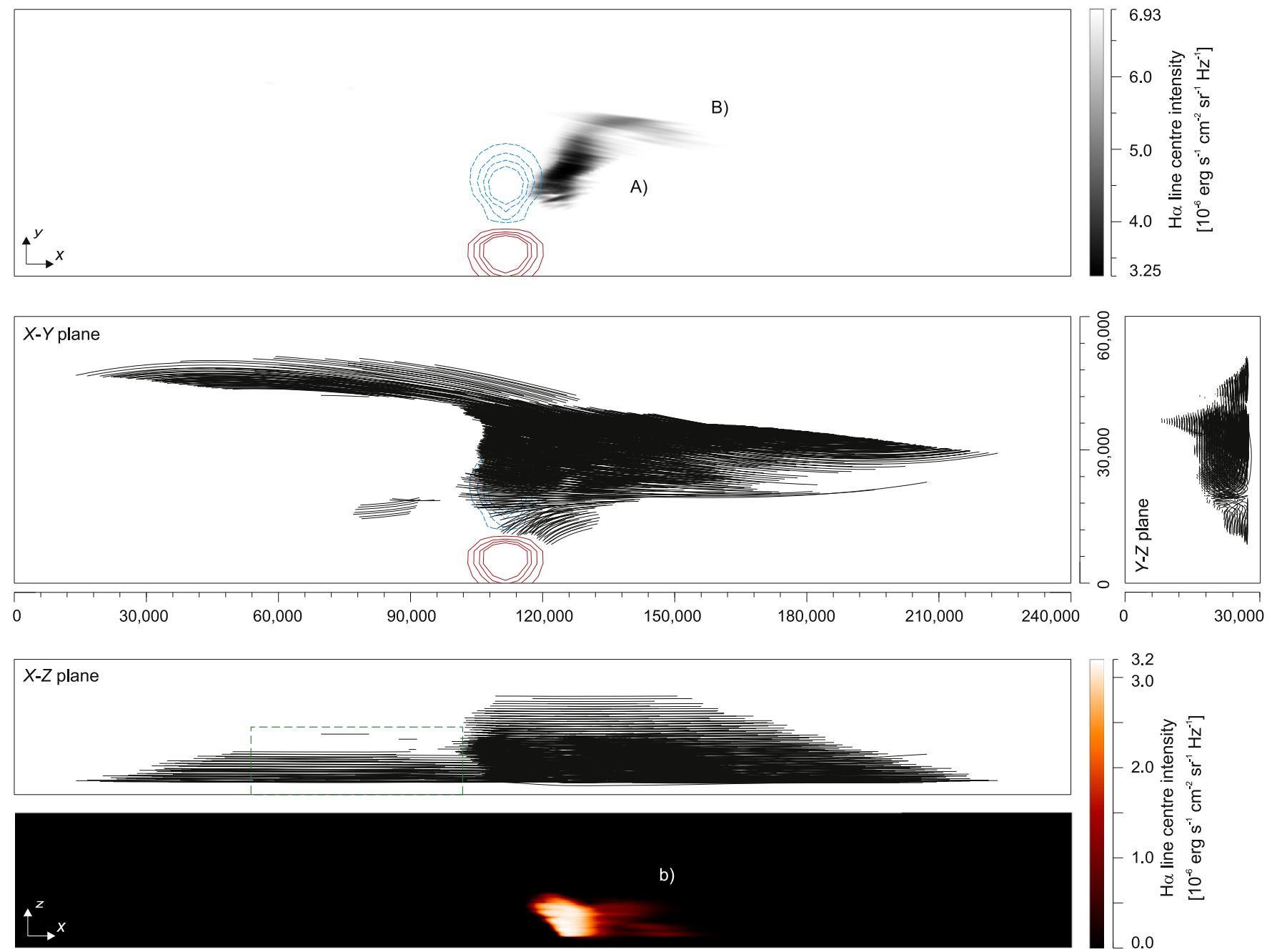

Figure 5. Snapshot 7. Same as in Figure 3. (A) denotes the darkest part of the filament composed of short dips formed due to the presence of the bipole. (b) and (B) highlight the position of the right part of the main body faintly visible in the prominence and the filament, respectively. The green dashed-line rectangle marks the area shown enlarged in Figure 10.

configuration illustrated in terms of the plotted dipped portions of the field lines, much more significant and pronounced changes occur when the synthetic $\mathrm{H} \alpha$ images are considered. This is partly due to the fact that when considering field lines, shallow and deep dips are given an equal significance. When considering the synthetic $\mathrm{H} \alpha$ emission or absorption, the deep dips that contain the high-pressure, and thus high-density prominence plasma feature more significantly than the shallow dips with low-pressure plasma.

Through comparing the plotted field lines and the synthetic $\mathrm{H} \alpha$ images in Figures 3-6, it is interesting to note that the major observable changes may often occur at locations where there is no noticeable larger-scale reconfiguration of the magnetic dips. As an example we can point out the apparent disappearance of the left $(\mathrm{c}, \mathrm{C})$ and later the right $(\mathrm{b}, \mathrm{B})$ part of the prominence main body in SNAPSHOTS 4 and 7, and their reappearance in SNAPSHOT 10. In Figures 3-5 one can see that the dipped magnetic field lines do not disappear or change dramatically, while at the same time the prominence plasma in the synthetic $\mathrm{H} \alpha$ images become invisible. Later in SNAPSHOT 10 (Figure 6), prominence plasma becomes visible again in both the prominence and filament synthetic $\mathrm{H} \alpha$ images. At the same time, the larger-scale configuration of the dipped magnetic field lines does not appear to change significantly between the snapshots. However, a detailed analysis of the plotted magnetic dips (see Figure 10) does reveal a combination of several small-scale changes of the magnetic field configuration. For instance, a larger number of dips is detectable at the location of the visible $\mathrm{H} \alpha$ emission in SNAPSHOT 10 compared to those in SnAPSHOT 7. This can be caused by either the creation of dips along previously undipped magnetic field lines or by the shifting of the central bottom parts of dips into the region of the visible $\mathrm{H} \alpha$ emission. Such small-scale localized changes of the magnetic field configuration may not be noticeable when studying the wholeprominence magnetic field. However, they can have a significant effect on the visibility of the prominence plasma.

Another effect that may contribute to the changes of the $\mathrm{H} \alpha$ visibility is a localized deepening or shallowing of the magnetic dips caused by the distortion of the magnetic field configuration. Even a small change of the dip depth by a few hundred kilometers results in a significantly different pressure in the hydrostatic prominence plasma (see Gunár et al. 2013). This can either mean a lower pressure caused by a dip becoming shallower (dip depth decreases) or a higher pressure if a dip becomes deeper. In the case where a dip becomes very shallow, 

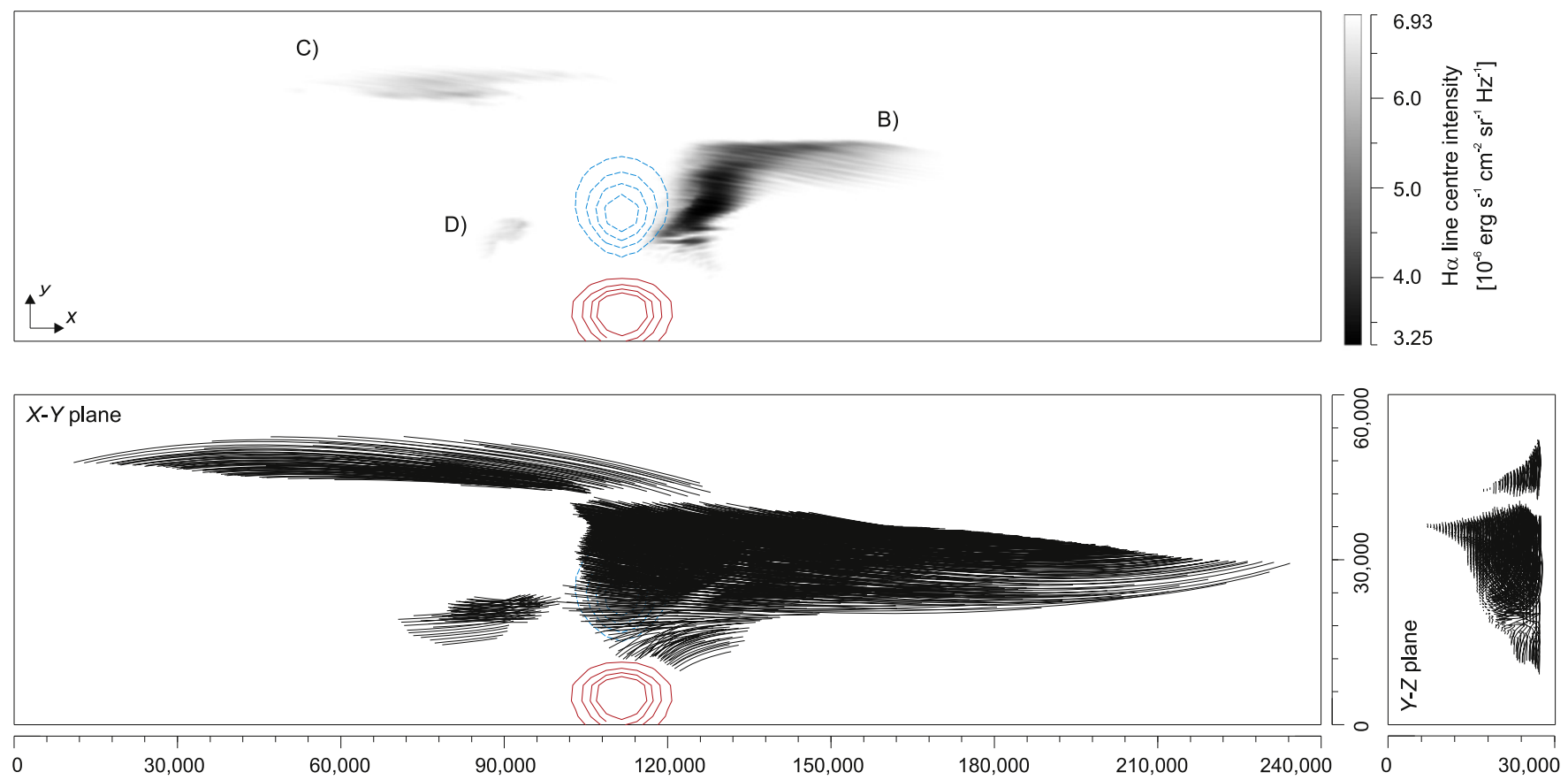

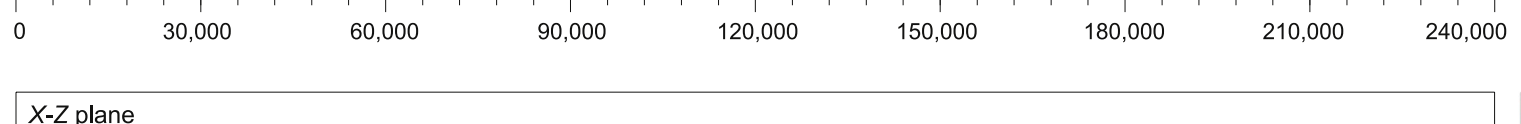

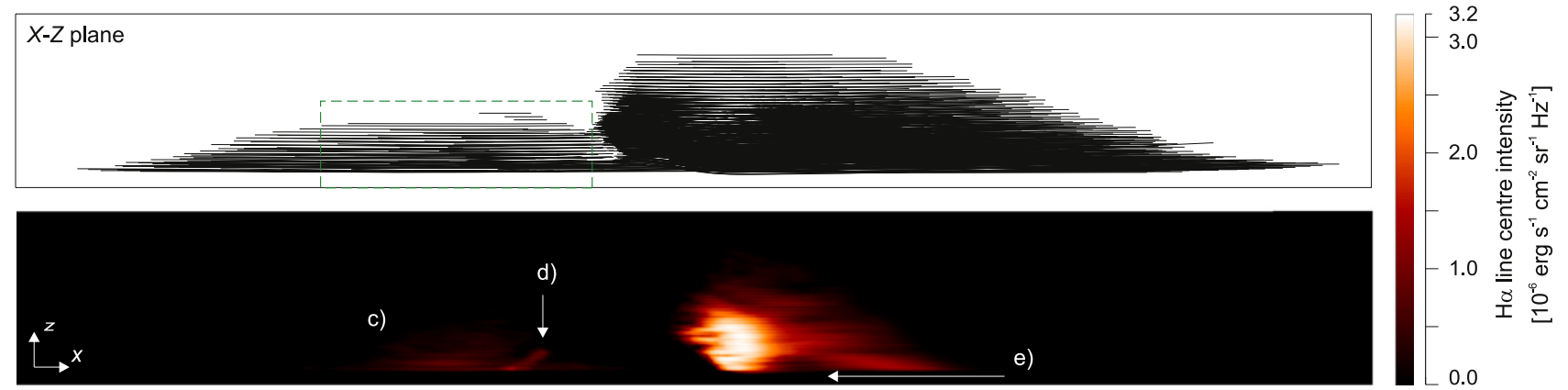

Figure 6. SnAPShot 10. Same as in Figure 3. (B) highlights the position of the right part of the main body visible in the filament. (c) and (C) highlight the position of the left part of the main body now faintly visible in the prominence and the filament, respectively. (d) and (D) denote a small region of short dips forming a quasivertical feature visible in the prominence. (e) highlights a darker arch-like region devoid of the bright emission. The green dashed-line rectangle marks the area shown enlarged in Figure 10.

the resulting low-pressure, and thus low-density plasma, does not absorb or scatter the background disk radiation effectively enough to be detectable in the $\mathrm{H} \alpha$ line, even if it is at the cool prominence-core temperatures. While it may not be visible in $\mathrm{H} \alpha$, the prominence material present in such a dip could in principle be detectable in different, optically thicker spectral lines, such as Ly $\alpha$ or He II $304 \AA$. On the other hand, if a dip depth increases, a higher pressure and thus higher density plasma will be more efficient in absorbing or scattering of the background radiation, thus increasing its $\mathrm{H} \alpha$ visibility. This could explain why the prominences and filaments appear to have different shapes when observed at different times or wavelengths. Here we note that while the magnetic dips shown in Figure 10 seem to be generally deeper in SNAPSHOT 10 than in SNAPShot 7, the considered configuration of the WPFS model does not allow us to clearly disentangle between individual causes of the local $\mathrm{H} \alpha$ visibility changes. In the future we will study the effect of the varying dip depth on the $\mathrm{H} \alpha$ visibility more systematically.

The described effect of the disappearance and reappearance of parts of the simulated prominence may offer an explanation for a part of the observed prominence fine structure dynamics, where individual fine structures or clusters of them disappear and reappear over time. Our results indicate that this may be caused by various small-scale changes of the magnetic dips due to the distortion of the entire magnetic field configuration. The latter is itself caused by the evolution of the photospheric flux distribution or perhaps by passing waves. We note that the 3D WPFS model we use is not suitable for tracking the changes of individual field lines between subsequent evolution steps. We are also unable to follow the dynamical evolution of the plasma of particular fine structures over time.

In all synthetic $\mathrm{H} \alpha$ images the main body of the prominence appears as a rather diffuse faint region both as a prominence and a filament. The brightest part of the prominence and the darkest part of the filament are formed by the barb. This is a consequence of the fact that the barb consists of shorter and deeper magnetic dips, probably due to its proximity to the bipole. In contrast, the initial arcade configuration forming the prominence main body consists of long but shallow dips that do not contain high-pressure, high-density hydrostatic prominence plasma. While this is true for the configuration considered here, which is a weakly twisted flux rope, if a more strongly twisted flux rope is used for the main body, then 

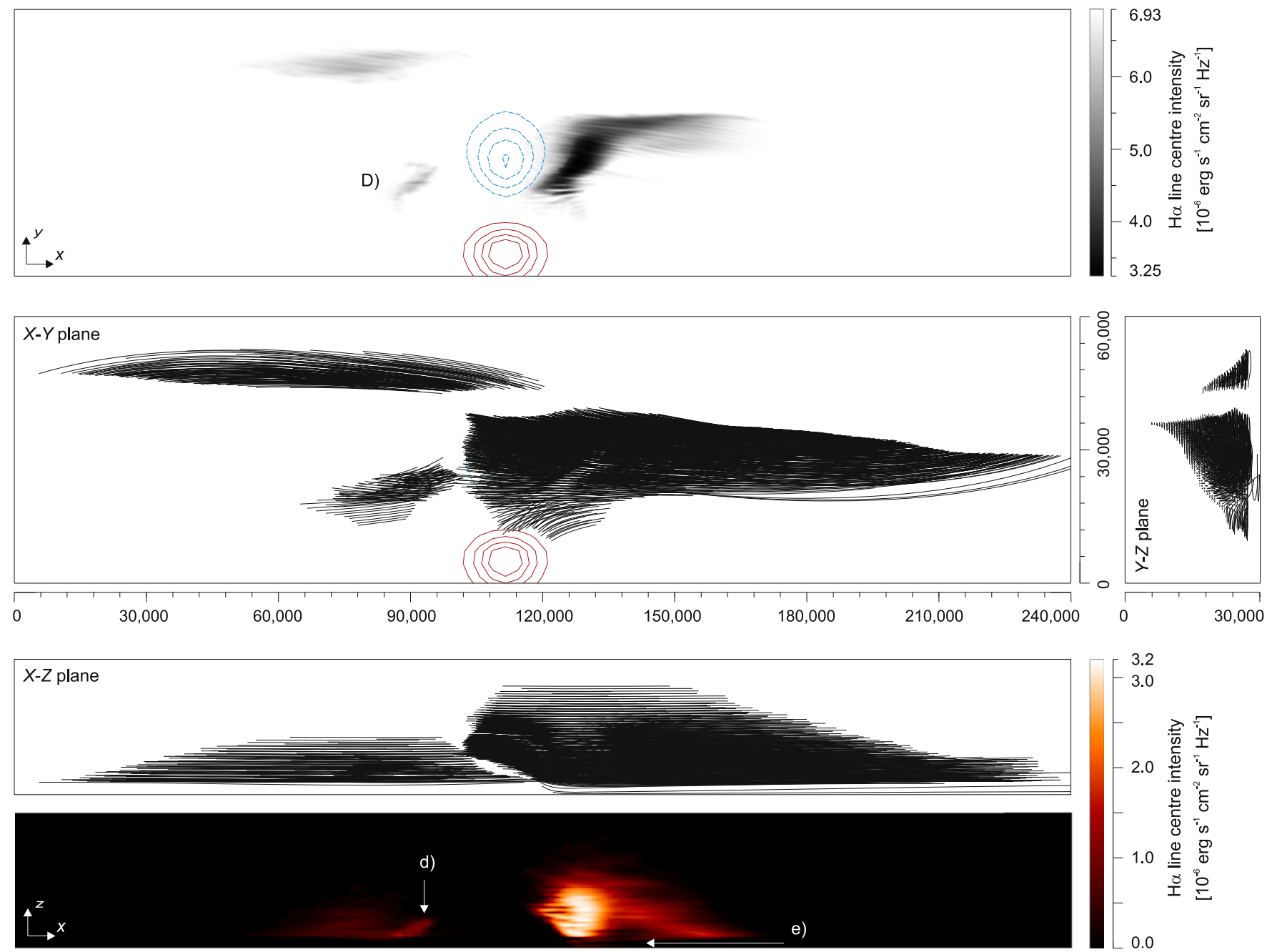

Figure 7. SNAPSHot 13. Same as in Figure 3. (d) and (D) denote a small region of short dips forming a quasi-vertical feature visible in the prominence. (e) highlights a darker arch-like region devoid of the bright emission.

the main body may be more visible. However, in the images of the plotted magnetic dips the main body is the most dominant feature throughout the whole studied evolution sequence. It takes up a much larger volume than the dips forming the barb and it is much more extended than the visible $\mathrm{H} \alpha$ intensity in the synthetic prominence and filament images. This is despite the fact that the plotted field lines in Figures 3-9 represent only the portions of the magnetic dips that can in principle be filled with plasma, not necessarily the whole extend of the dips (see Gunár et al. 2013). The difference between the simulated $\mathrm{H} \alpha$ images and the plotted field lines is a result of the fact that a large portion of each filled dip contains plasma with low PCTR temperatures. These PCTR temperatures are in our case rising up to $100,000 \mathrm{~K}$ but in principle can be even higher. Such PCTR plasma cannot be detected in the $\mathrm{H} \alpha$ line, but could be visible in hotter spectral lines. In such a case it could form a diffuse halo around the central cool parts of prominences, as discussed by Gunár et al. (2014b).

In the series of prominence evolution steps presented in this paper (SNAPSHOTS 7 to 19) we can observe the creation of an arch-like dark region located below the prominence (marked as (e) in Figure 1). Such a region, often called a bubble, is a source of controversy. These bubbles were reported by Berger et al. $(2010,2011)$ and studied by, for example, Dudík et al.
(2012) and Gunár et al. (2014b). For the prominence studied in the present paper the formation of the arch-like region is an unexpected result. We have not attempted to modify the photospheric flux distribution or the LOS direction to specially create favorable conditions leading to its appearance. The arclike feature is caused by the disruption of the initial arcade magnetic field configuration by the passing minority bipole and it appears only in the particular view we have used. It may not be visible in a significantly different view. This dark void under the prominence is a location of shallow magnetic dips that contain only low-pressure, low-density prominence plasma that does not produce detectable synthetic $\mathrm{H} \alpha$ intensity. However, many magnetic dips are located along any given LOS traversing the dark void (see Figures 5-9) and these contain prominence material that can in principle be visible in different, optically thicker spectral lines. This is consistent with the conclusions of Gunár et al. (2014b). Interestingly, such an archlike region is not evident in Mackay \& van Ballegooijen (2009, see their Figure 5), who studied the same prominence evolution sequence but used a visualization method based only on the drawing of the position of the magnetic dips. In agreement with this, it cannot be detected in the plots showing the dipped portions of the magnetic field lines in Figures 6-9. 

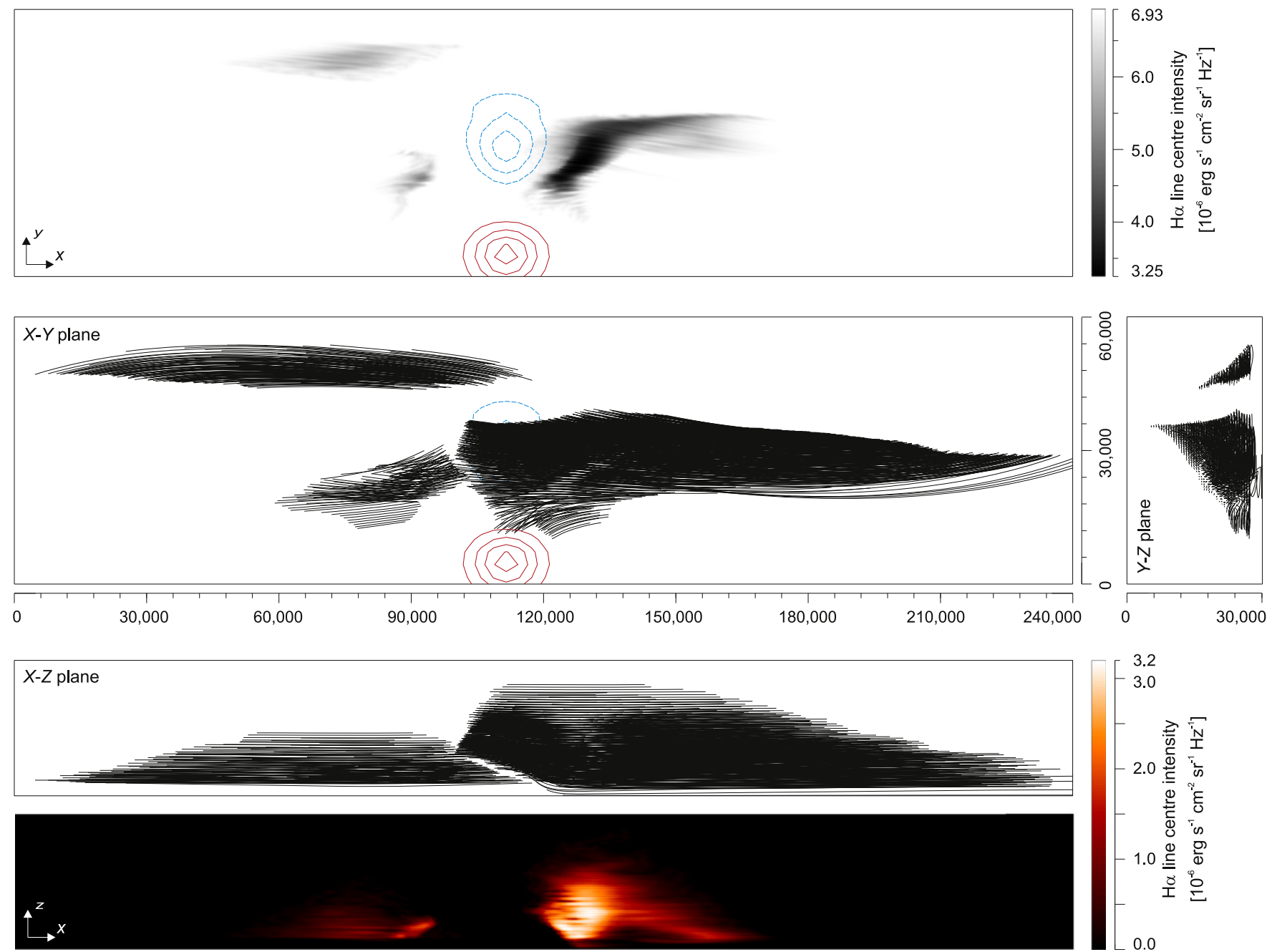

Figure 8. SnAPShot 16. Same as in Figure 3.

Another interesting prominence fine structure feature that has formed and evolves naturally is the small quasi-vertical region on the left of the bipole (marked as (d) and (D) in Figures 1 and 2 , respectively). This consists of a series of short and relatively deep dips that propagate semi-vertically (see the red field lines shown in Figure 10). These form a feature akin to the often observed vertical thread-like prominence fine structures. However, the observed vertical threads have smaller overall dimensions than the modeled quasi-vertical feature produced in the present paper. A mechanism that forms pile-ups of magnetic dips, one above each other, is assumed also to be responsible for the creation of the observed vertical thread-like fine structures (see, e.g., Heinzel \& Anzer 2006; Gunár et al. 2013b).

The tilt of the central bright region in the synthetic $\mathrm{H} \alpha$ prominence images between SNAPSHOTS 1 and 19 is gradually changing from the left to the right. This fact is interesting because it could indicate a rotational motion of a large part of the prominence. However, in this study we do not assume any rotational movement in the photospheric flux distribution. Rather, the gradual change of the tilt results from the combination of the actual alignment of individual dips in the magnetic field configuration distorted by the shifting minority polarity and the assumed viewing angle. The perceived orientation of the central bright region could be expected to be different in a case of a significantly different viewing angle.

All significant changes in the modeled magnetic field configuration result from the fact that the inserted bipole has a strong distorting effect on the initial arcade configuration. Although we assume a typical flux of the minority bipole that is much weaker than the overall flux of the arcade, only a small portion of the arcade flux actually belongs to the region with the dipped magnetic field lines and thus to the prominence itself. In fact, the bipole flux is nearly three times stronger than the initial filament flux (see Section 2.1 and Mackay \& van Ballegooijen 2009). That means that even relatively small changes of the position of the bipole in the photosphere can have a strong influence on the magnetic field structure above. Such an effect can be expected on the Sun, where the photospheric magnetic flux distributions are even more complex and dynamical than the one we consider in this study. The complex evolving photospheric flux distributions combined with ongoing flux emergence or cancellation events could have an even more profound influence on the evolution and dynamics of prominences and their fine structures such as we have demonstrated here.

Finally we note that in this work we do not assume any distortion of the magnetic field configuration due to the presence of the prominence plasma in the magnetic dips (we 

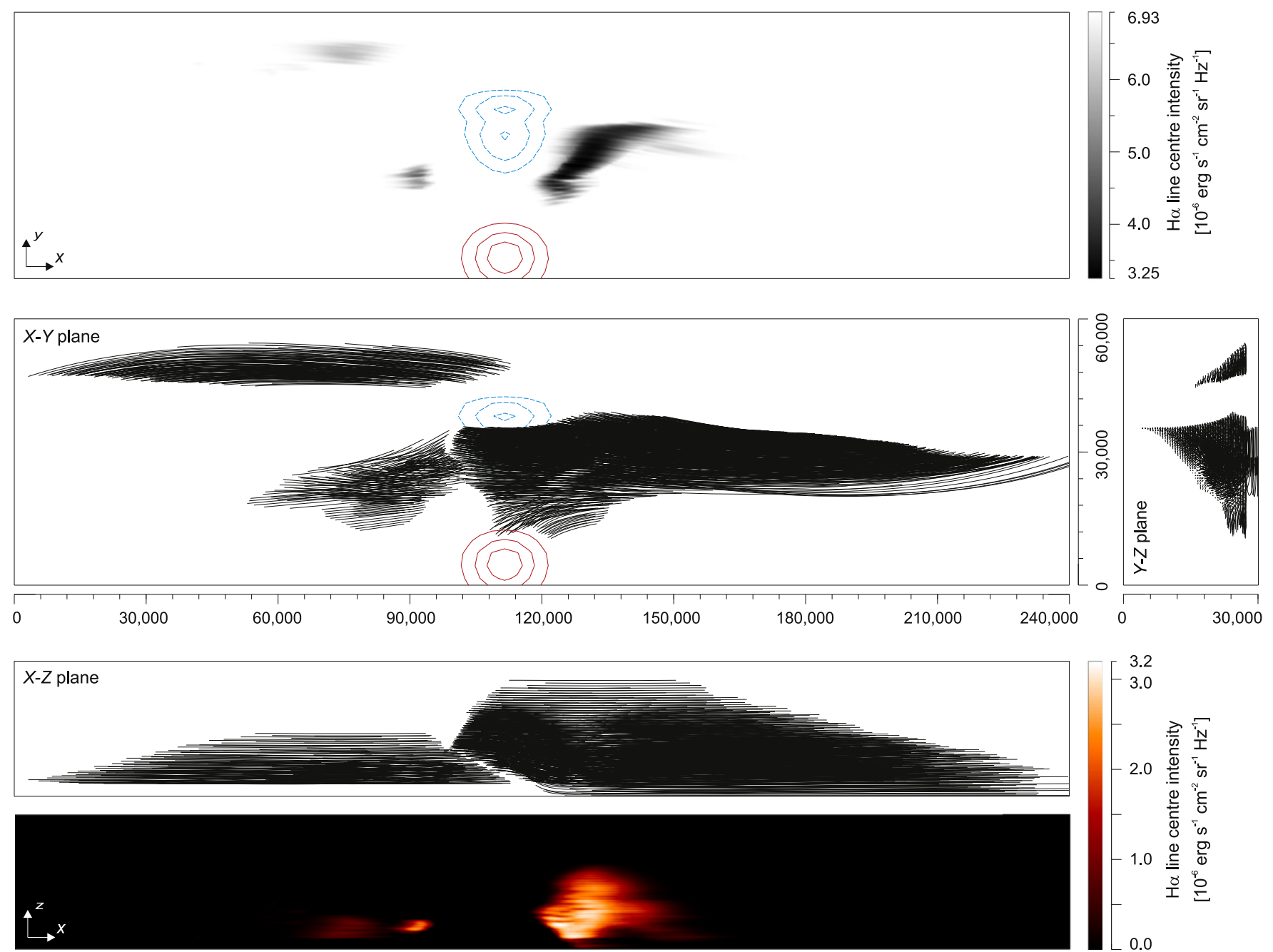

Figure 9. Snapshot 19. Same as in Figure 3.

assume a low- $\beta$ plasma). If the prominence plasma would have a distorting effect on the magnetic field configuration then even more small-scale dynamical behavior of the prominence fine structures could be expected. We will consider this in the future.

\section{CONCLUSIONS}

We have shown that the brightest part of the prominence and the darkest part of the filament consistently correspond to the region of relatively short and deep magnetic dips branching out from the main prominence body toward the inserted bipole. These dips form a barb where, due to the larger depth, dips contain plasma with a higher pressure and higher density compared to the shallower dips forming the main body. The barb is visible as a compact central bright quasi-vertical feature in the synthetic prominence $\mathrm{H} \alpha$ images (Figure 1), while the main body produces a diffuse weaker emission around it. This means that often the most easily identifiable parts of observed prominences may belong to barbs, and as a consequence have a more complex magnetic topology. This fact should be taken into account when prominence observations are analyzed, especially when the prominence magnetic field is being inferred from spectro-polarimetric observations.
Following the evolution of the modeled prominence we see the creation of an arch-like region with a dark void (bubble) underneath the bright prominence. This region is created naturally without our intentional modification of the underlying photospheric flux distribution or the direction of the LOS. It is caused by the presence of the bipole disrupting the initial arcade magnetic field configuration and it evolves over several subsequent snapshots (Figures 6-8). Interestingly, such an arch-like region cannot be identified in the images of the plotted magnetic dips. At its location we can clearly see numerous dips along any given LOS passing through the dark void. However, these dips are shallow and do not contain hydrostatic prominence plasma with sufficient pressure (and thus density) to produce detectable synthetic $\mathrm{H} \alpha$ intensity. Nevertheless, they are still filled with low-density plasma with temperatures ranging from, in our case, 7000 to $100,000 \mathrm{~K}$. Therefore, this region may emit radiation that could be detectable in spectral lines that are optically thicker than the $\mathrm{H} \alpha$ line.

We also see the creation of a small-scale quasi-vertical feature formed by a pile-up of short dips one above each other. This resembles the often observed vertical thread-like fine structures. However, we note that the modeled feature produced here has larger overall dimensions than those usually observed. However, this example shows that basic conditions 


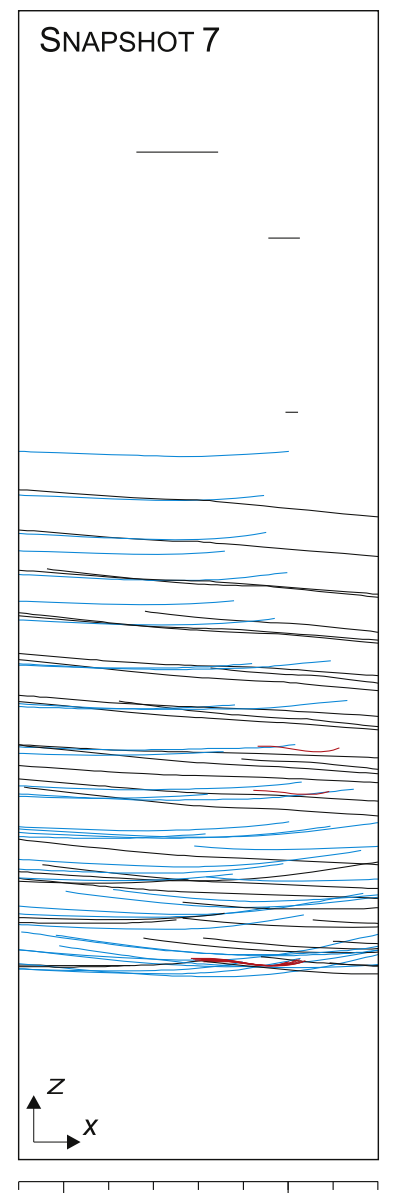

60,000

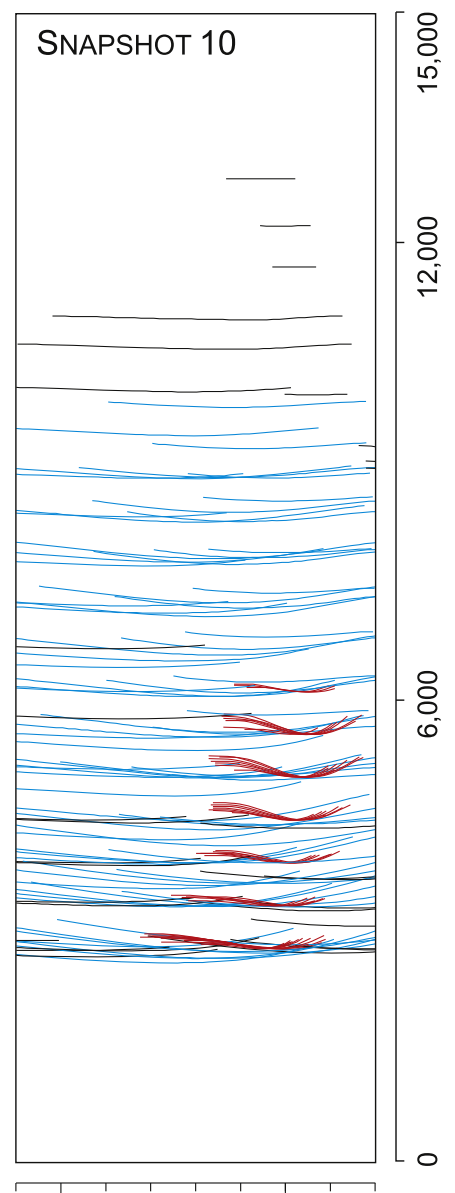

60,000
90,000

Figure 10. Enlarged view of the $x-z$ plane showing the dipped portions of filed lines. This view corresponds to the area marked by green dashed-line rectangles shown in Figures 5 and 6, respectively. The $z$-axis dimension is increased tenfold to allow for depth of the magnetic dips to be discernible. The blue and black field lines belong to the left part of the main body (c, C). The blue color marks the field lines where the bottom of the dip is present in the selected view. Red field lines belong to the region of short dips on the left side of the bipole $(\mathrm{d}, \mathrm{D})$. Dimensions are in kilometers.

for the formation of such vertical threads can occur naturally in the local configurations of the prominence magnetic field. For cases where the prominence plasma has a distorting effect on the magnetic field (high- $\beta$ plasma) such pile-ups of dips would form more easily due to the vertical propagation of the deformation of the individual magnetic field lines.

Through the present study we show that no simple relationship exists between the structure of the prominence magnetic field and the observable $\mathrm{H} \alpha$ emission or absorption of the cool prominence plasma. While magnetic field lines provide the basic skeleton of prominences, they are not always representative of the visible $\mathrm{H} \alpha$ structures of prominences and filaments. Very different features may be seen in the $\mathrm{H} \alpha$ images of both prominences and filaments compared to the plots of the dipped portions of the magnetic field lines. Varying features include different larger-scale structures, different spatial distributions of the individual fine structures, the presence of regions devoid of the visible $\mathrm{H} \alpha$ emission or absorption but still filled with dipped magnetic field lines, and the creation of vertical $\mathrm{H} \alpha$ features out of horizontal magnetic fields. In addition to this, very different variations may occur in

the simultaneous $\mathrm{H} \alpha$ images of prominences and filaments, where one or the other may change significantly while the other remains the same.

In summary, the presented study of the simultaneous prominence and filament evolution shows that many puzzling aspects of prominences may be explained by studying the link between the evolution of the photospheric magnetic flux distribution, the prominence magnetic field configuration, and the prominence plasma along with its visibility in synthetic $\mathrm{H} \alpha$ images. These aspects include:

1. the formation of dark voids (bubbles) seen in the prominence $\mathrm{H} \alpha$ images but not in the plots of the dipped magnetic field lines;

2. the formation of quasi-vertical structures visible in the prominence $\mathrm{H} \alpha$ images inside the horizontal magnetic field configurations;

3. the disappearance and reappearance of large parts of $\mathrm{H} \alpha$ prominences and filaments while the magnetic field structure seems nearly unchanged;

4. visible variations of the tilt of the $\mathrm{H} \alpha$ prominence structures without significant change of the magnetic field structure or any rotational movement; and

5. the simultaneous presence of horizontally aligned $\mathrm{H} \alpha$ fine structures in absorption viewed as a filament corresponding to vertical $\mathrm{H} \alpha$ structures in emission viewed as a prominence.

The present paper demonstrates that 3D models of entire prominences with their numerous fine structures are very useful for understanding many aspects of prominence nature. The best results can be achieved by combining detailed prominence magnetic field simulations together with a realistic prominence plasma description and the radiative transfer modeling that provides the synthetic emission of the modeled prominence plasma. These models, such as the 3D WPFS model developed by Gunár \& Mackay (2015) and used here, represent a stepping stone toward 3D models of actual observed prominences driven by the observed photospheric flux distributions. Such models will be considered in the future. In future studies we will also concentrate on the mass of the modeled prominence and its variation. Through doing so we will also study the distribution of the plasma parameter $\beta$ in the modeled prominence and its variation throughout the evolution sequence.

S.G. acknowledges support from the European Commission via the Marie Curie Actions-Intra-European Fellowships Project No. 328138. D.H.M. acknowledges financial support from the STFC, the Leverhulme Trust, and NASA. The simulations in the present paper use the STFC funded UKMHD supercomputer in St Andrews.

\section{REFERENCES}

Aulanier, G., DeVore, C. R., \& Antiochos, S. K. 2006, ApJ, 646, 1349 Ballester, J. L. 2014, in IAU Symp. 300, Nature of Prominences and Their Role in Space Weather, ed. B. Schmieder, J.-M. Malherbe \& S. T. Wu (Paris: IAU), 30

Berger, T. 2014, in IAU Symp. 300, Nature of Prominences and Their Role in Space Weather, ed. B. Schmieder, J.-M. Malherbe \& S. T. Wu (Paris: IAU), 15

Berger, T., Testa, P., Hillier, A., et al. 2011, Natur, 472, 197

Berger, T. E., Shine, R. A., Slater, G. L., et al. 2008, ApJL, 676, L89

Berger, T. E., Slater, G., Hurlburt, N., et al. 2010, ApJ, 716, 1288

Casini, R., López Ariste, A., Paletou, F., \& Léger, L. 2009, ApJ, 703, 114

David, K.-H. 1961, ZAp, 53, 37 
DeVore, C. R., \& Antiochos, S. K. 2000, ApJ, 539, 954

Dudík, J., Aulanier, G., Schmieder, B., Zapiór, M., \& Heinzel, P. 2012, ApJ, 761,9

Galsgaard, K., \& Longbottom, A. W. 1999, ApJ, 510, 444

Gunár, S. 2014, in IAU Symp. 300, Nature of Prominences and Their Role in Space Weather, ed. B. Schmieder, J.-M. Malherbe \& S. T. Wu (Paris: IAU), 59

Gunár, S., Heinzel, P., Anzer, U., \& Mackay, D. H. 2013b, JPhCS, 440, 012035 Gunár, S., \& Mackay, D. H. 2015, ApJ, 803, 64

Gunár, S., Mackay, D. H., Anzer, U., \& Heinzel, P. 2013, A\&A, 551, A3

Gunár, S., Mein, P., Schmieder, B., Heinzel, P., \& Mein, N. 2012, A\&A, 543, A93

Gunár, S., Schwartz, P., Dudík, J., et al. 2014b, A\&A, 567, A123

Heinzel, P., \& Anzer, U. 2006, ApJL, 643, L65

Heinzel, P., Gouttebroze, P., \& Vial, J.-C. 1994, A\&A, 292, 656

Heinzel, P., Gunár, S., \& Anzer, U. 2015a, A\&A, 579, A16

Heinzel, P., Schmieder, B., Mein, N., \& Gunár, S. 2015b, ApJL, 800, L13

Hillier, A., Berger, T., Isobe, H., \& Shibata, K. 2012, ApJ, 746, 120

Kosugi, T., Matsuzaki, K., Sakao, T., et al. 2007, SoPh, 243, 3

Labrosse, N., Heinzel, P., Vial, J., et al. 2010, SSRv, 151, 243
Leroy, J. L. 1989, in Dynamics and Structure of Quiescent Solar Prominences, Vol. 150, ed. E. R. Priest (Dordrecht: Kluwer)

Lionello, R., Mikić, Z., Linker, J. A., \& Amari, T. 2002, ApJ, 581, 718

Mackay, D. H., \& Gaizauskas, V. 2003, SoPh, 216, 121

Mackay, D. H., Karpen, J. T., Ballester, J. L., Schmieder, B., \& Aulanier, G. 2010, SSRv, 151, 333

Mackay, D. H., \& van Ballegooijen, A. A. 2005, ApJL, 621, L77

Mackay, D. H., \& van Ballegooijen, A. A. 2006, ApJ, 641, 577

Mackay, D. H., \& van Ballegooijen, A. A. 2009, SoPh, 260, 321

Martens, P. C., \& Zwaan, C. 2001, ApJ, 558, 872

Orozco Suárez, D., Asensio Ramos, A., \& Trujillo Bueno, J. 2014, A\&A, 566, A46

Schmieder, B., Chandra, R., Berlicki, A., \& Mein, P. 2010, A\&A, 514, A68

Tsuneta, S., Ichimoto, K., Katsukawa, Y., et al. 2008, SoPh, 249, 167

van Ballegooijen, A. A., \& Martens, P. C. H. 1989, ApJ, 343, 971

van Ballegooijen, A. A., Priest, E. R., \& Mackay, D. H. 2000, ApJ, 539, 983

Xia, C., Chen, P. F., \& Keppens, R. 2012, ApJL, 748, L26

Xia, C., Chen, P. F., Keppens, R., \& van Marle, A. J. 2011, ApJ, 737, 27 